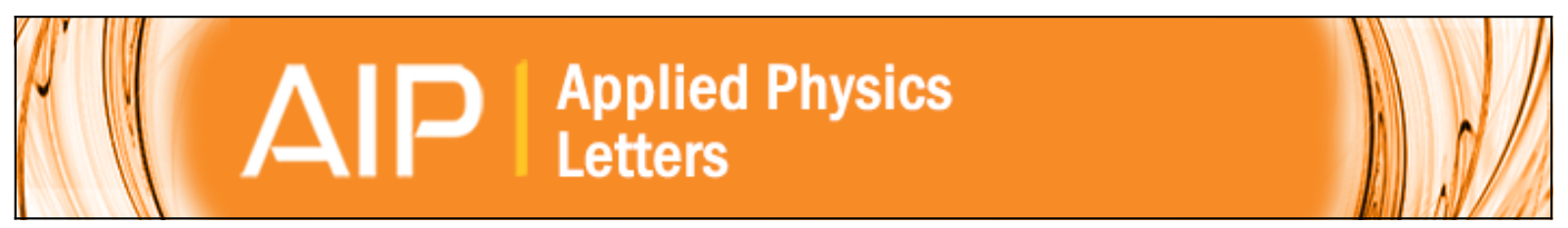

\title{
Band energy control of molybdenum oxide by surface hydration
}

Keith T. Butler, Rachel Crespo-Otero, John Buckeridge, David O. Scanlon, Edward Bovill, David Lidzey, and Aron Walsh

Citation: Applied Physics Letters 107, 231605 (2015); doi: 10.1063/1.4937460

View online: http://dx.doi.org/10.1063/1.4937460

View Table of Contents: http://scitation.aip.org/content/aip/journal/apl/107/23?ver=pdfcov

Published by the AIP Publishing

\section{Articles you may be interested in}

Surface oxidation energetics and kinetics on MoS2 monolayer

J. Appl. Phys. 117, 135301 (2015); 10.1063/1.4916536

First principles study on InP $(001)-(2 \times 4)$ surface oxidation

J. Appl. Phys. 113, 103705 (2013); 10.1063/1.4794826

A theoretical investigation on photocatalytic oxidation on the $\mathrm{TiO} 2$ surface

J. Chem. Phys. 136, 024706 (2012); 10.1063/1.3676261

First-principles study of GaAs(001)- $\beta 2$ ( $2 \times 4$ ) surface oxidation and passivation with $\mathrm{H}, \mathrm{Cl}, \mathrm{S}, \mathrm{F}$, and $\mathrm{GaO}$ J. Appl. Phys. 107, 103720 (2010); 10.1063/1.3369540

Electronic structure of coordinatively unsaturated molybdenum and molybdenum oxide carbonyls J. Chem. Phys. 130, 064301 (2009); 10.1063/1.3073855

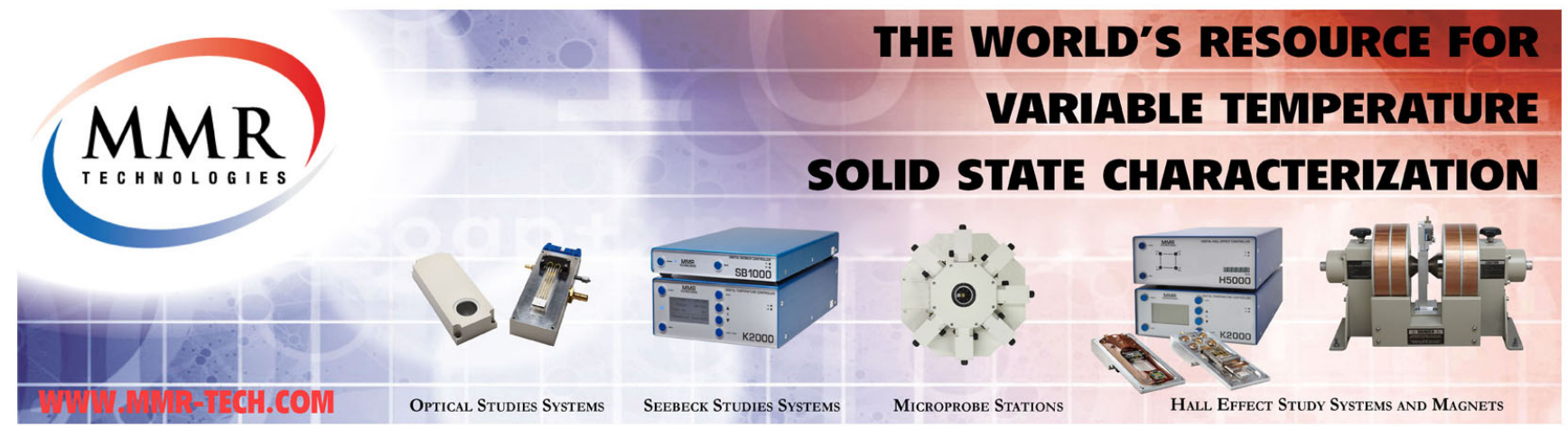




\title{
Band energy control of molybdenum oxide by surface hydration
}

\author{
Keith T. Butler, ${ }^{1, a)}$ Rachel Crespo-Otero, ${ }^{2}$ John Buckeridge, ${ }^{3}$ David O. Scanlon, ${ }^{3}$ \\ Edward Bovill, ${ }^{4}$ David Lidzey, ${ }^{4}$ and Aron Walsh ${ }^{1}$ \\ ${ }^{1}$ Department of Chemistry, University of Bath, Claverton Down, Bath BA2 7AY, United Kingdom \\ ${ }^{2}$ School of Biological and Chemical Sciences, Queen Mary University London, Mile End Road, \\ London E1 4NS, United Kingdom \\ ${ }^{3}$ University College London, Kathleen Lonsdale Materials Chemistry, 20 Gordon Street, London WC1H OAJ, \\ United Kingdom \\ ${ }^{4}$ Department of Physics and Astronomy, University of Sheffield, Hicks Building, Hounsfield Road, \\ Sheffield S3 7RH, United Kingdom
}

(Received 17 September 2015; accepted 27 November 2015; published online 10 December 2015)

\begin{abstract}
The application of oxide buffer layers for improved carrier extraction is ubiquitous in organic electronics. However, the performance is highly susceptible to processing conditions. Notably, the interface stability and electronic structure is extremely sensitive to the uptake of ambient water. In this study we use density functional theory calculations to asses the effects of adsorbed water on the electronic structure of $\mathrm{MoO}_{x}$, in the context of polymer-fullerene solar cells based on PCDTBT. We obtain excellent agreement with experimental values of the ionization potential for pristine $\mathrm{MoO}_{3}(010)$. We find that IP and EA values can vary by as much as $2.5 \mathrm{eV}$ depending on the oxidation state of the surface and that adsorbed water can either increase or decrease the IP and EA depending on the concentration of surface water. (C) 2015 AIP Publishing LLC.

[http://dx.doi.org/10.1063/1.4937460]
\end{abstract}

The application of high work function transition metal oxide (TMO) layers at electrode surfaces in organic photovoltaic (OPV) and organic light emitting diodes (OLEDs) has become extensive. This is due to the enhanced charge transport at the interfaces upon addition of thin layers of TMO; in particular, TMOs such as $\mathrm{MoO}_{3}, \mathrm{WO}_{3}$, and $\mathrm{V}_{2} \mathrm{O}_{5}$ have been applied as hole transport layers (HTLs). The addition of HTLs, as well as improvements in device encapsulation, mean that targets for efficiency and lifetime for OPV solar cells are now within reach; nonetheless, major challenges in device manufacture and fundamental questions regarding operating principles still remain to be answered.

One of the challenges faced by organic electronic devices is the efficient transfer of charge between the organic layer and the electrical contact. This has been an active field of research for two decades, and great effort has been invested both experimentally and theoretically into understanding the factors which dictate these processes. The basic problem would appear simple: efficient charge transport across the interface requires electron energy levels in the contact and organic layers to be well matched, to reduce barriers and achieve Ohmic behavior. ${ }^{1-4}$ Nevertheless, the $a$ priori identification of optimal organic/inorganic components remains extremely difficult. The application of TMO layers to improve this process has become a popular strategy with improved performance reported in organic field effect transistor, ${ }^{5} \mathrm{OLED},{ }^{6-8}$ and $\mathrm{OPV}^{9-11}$ devices. Indeed the application of inter-layers for improved electrical contacts is becoming an extremely popular strategy in both organic and inorganic semiconductor architectures. ${ }^{12,13}$ Various mechanisms have been proposed for this performance, including

${ }^{\text {a)} E l e c t r o n i c ~ m a i l: ~ k . t . b u t l e r @ b a t h . a c . u k ~}$ favorable energy-band alignment and p-type doping of the organic material by the TMO. ${ }^{14}$

$\mathrm{MoO}_{3}$ is an insulating/semiconducting TMO; structurally it is based on strongly distorted edge-sharing octahedra. The strong distortion of the octahedra leads to the formation of a layered structure. Molybdenum is in oxidation state (VI) and the electron configuration is $d^{0}$. Chemically $\mathrm{MoO}_{2}$ is also stable; the electronic $d^{2}$ configuration allows the formation of Mo-Mo bonds, resulting in metallic conduction. Both $\mathrm{MoO}_{3}$ and $\mathrm{MoO}_{2}$ have long been appreciated for their catalytic activity. ${ }^{15}$ In $\mathrm{MoO}_{3}$ the valence band edge is formed from $\mathrm{O}$ p-orbitals, while the conduction band minimum consists of the empty Mo d-orbitals. If the surface is reduced, the d-orbitals of Mo become occupied, altering the ionisation potential. The ionisation potential can also be altered by other surface modifications.

Recent studies into the effects of processing on the performance of devices featuring $\mathrm{MoO}_{3}$ HTLs have emphasised a number of critical parameters affecting device performance, primarily layer thickness, metal oxidation state, ${ }^{16,17}$ and processing atmosphere. ${ }^{18}$ It was reported that processing in air has a large detrimental effect on device characteristics unless the $\mathrm{MoO}_{3}$ layer was annealed prior to exposure and the role of adsorbed water was invoked to explain the observed changes. The effects of exposure over time were previously also shown to result in a realignment of $\mathrm{MoO}_{x}$ energy levels and a loss of doping activity. ${ }^{19}$ Several possible roles of water have been suggested, from surface hydration or hydroxylation, modifying electron energies through the formation of dipole layers, ${ }^{20}$ to hydrolysis of $\mathrm{MoO}_{3}$ layers into smaller crystallites. ${ }^{19}$

Density functional theory (DFT) is a useful tool for probing the influence of surfaces on electron energies. Recently we demonstrated how modification of the surface 
of $\mathrm{SnO}_{2}$ can tune the band energies to obtain high work functions required for organic electronic applications. ${ }^{21,22}$ Monolayers of organic molecules can also have dramatic effects on the work function, even at low coverage. ${ }^{23}$ DFT has also previously been applied to study the electronic structure of bulk $\mathrm{MoO}_{3}{ }^{24}$ and $\mathrm{MoO}_{3}$ surfaces ${ }^{25}$ and interfaces of $\mathrm{MoO}_{3}$ and organic semiconductors; ${ }^{17}$ however, the effect of the ambient water on the energy band levels has hitherto been neglected.

In this study we investigate how surface modifications caused by atmospheric exposure affect the electronic energy levels of $\mathrm{MoO}_{3}$, considering several possible effects of water on the $\mathrm{MoO}_{3}$ layer. We begin by quantifying the effect of the surface contribution, demonstrating how altering the surface oxidation state can shift the electron energies by $\sim 2.5 \mathrm{eV}$. We then assess how adsorption of water affects the ionization potential (IP) and EA, and geometry of adsorption and electron energies are calculated as a function of water coverage. Finally we also consider further possible effects of water, such as surface hydroxylation and HTL cleavage.

All electronic structure calculations were preformed using the Vienna Ab Initio Simulation Package (VASP) ${ }^{26}$ within the projector augmented wave formalism. ${ }^{27,28}$ The $\mathrm{MoO}_{3}$ crystal structure was obtained from experimental coordinates. ${ }^{29}$ The coordinates were fully relaxed for all degrees of freedom using the PBESol functional ${ }^{30}$ a plane wave cutoff energy of $500 \mathrm{eV}$ and $k$-point density was scaled to the unit cell to achieve a uniform sampling with a cutoff density of $0.04 \AA$ in reciprocal space as prescribed by Moreno and Soler. ${ }^{31}$ From the relaxed coordinates, the electronic band structure was calculated using the hybrid HSE06 functional. ${ }^{32}$ These coordinates also used to cleave surface slabs. The slabs were cleaved along the $(010)$ plane. The IPs were calculated as the difference between the valence band maximum (excluding the influence of surface states) and the vacuum Hartree potential in the calculation. The valence band maximum excluding surface states is obtained by aligning the core eigenstates of the Mo ions at the centre of the slab to the eigenstates calculated for the pure bulk. Further details of this procedure can be found elsewhere. ${ }^{4}$ The Hartree potential was analysed using the open-source MacroDensity package. ${ }^{33}$

We have collated the calculated electronic energies in an energy-band-diagram (Figure 1) with the highest occupied molecular orbital (HOMO) energy of a common p-type organic semiconductor (PCDTBT) and the band energies of the widely used electrode material, $\mathrm{Sn}$ doped $\mathrm{In}_{2} \mathrm{O}_{3}$ (ITO). The values calculated for pristine $\mathrm{MoO}_{3}(\mathrm{IE}=9.66 \mathrm{eV}$, $\mathrm{EA}=6.96 \mathrm{eV}$ ) compare well with the experientially obtained values $(\mathrm{IE}=9.68 \mathrm{eV}, \mathrm{EA}=6.7 \mathrm{eV}) .{ }^{34}$

We begin by considering how the surface influences the IP and EA. Following the formalism of Bardeen ${ }^{35}$ it is possible to separate the slab IP into bulk and surface contributions

$$
I P=I P_{\text {bulk }}+\Delta V_{\text {surf }},
$$

where the $\Delta V_{\text {surf }}$ term arises from the spilling of electrons out from the surface ${ }^{36,37}$ and the $\mathrm{IP}_{\text {bulk }}$ term is largely determined by the crystal environment. ${ }^{38}$

The surface dipole contribution to the IP of $\mathrm{MoO}_{3}$ is calculated by considering the difference between the surface

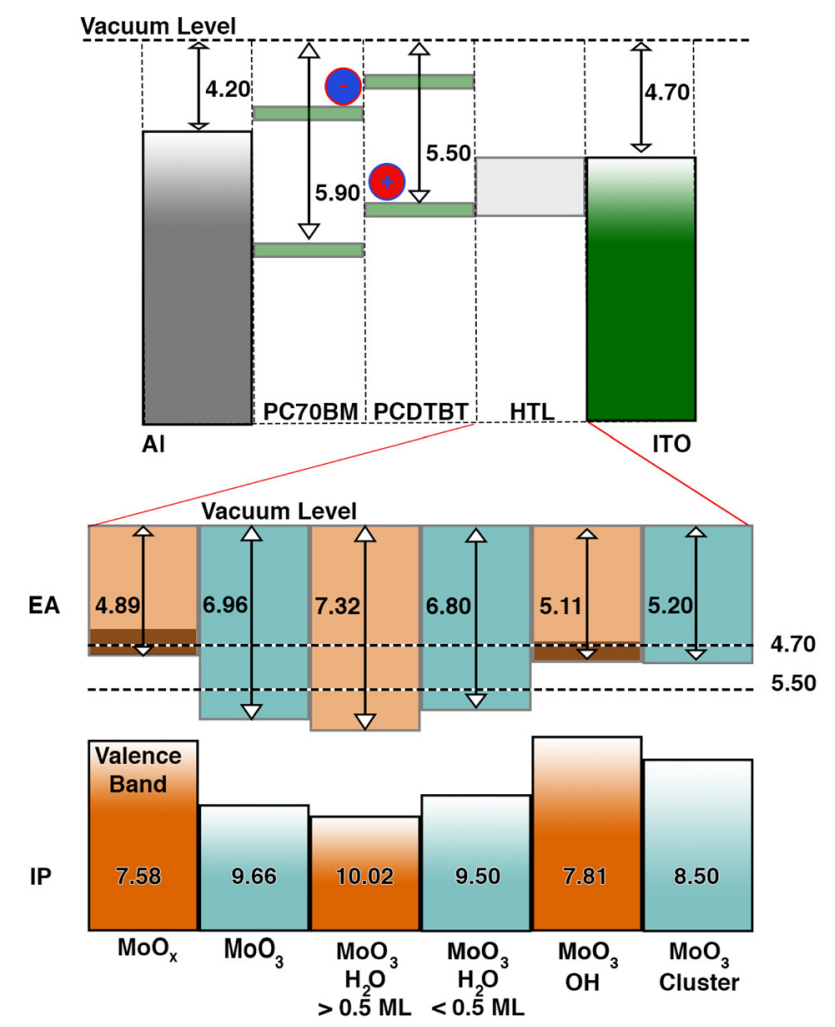

FIG. 1. Energy-band-alignment diagram of $\mathrm{MoO}_{x}$ for varying states of surface oxidation and hydration with PCDTBT and $\mathrm{Sn}: \mathrm{In}_{2} \mathrm{O}_{3}$ (ITO). The lower panel shows energy levels aligned through the vacuum levels of the materials and the electron affinities (EA) and ionisation potentials (IP) are presented for each system. The various scenarios in the bottom panel can occupy the HTL in the architecture of the upper panel. $\mathrm{MoO}_{x}$ has stoichiometry $\mathrm{MoO}_{2.8}$. In $\mathrm{MoO}_{x}$ and $\mathrm{MoO}_{3} \mathrm{OH}$ the shaded regions in the conduction band indicate occupation by excess electrons. The values for the $\mathrm{MoO}_{3}$ clusters are measurements from Ref. 16. All energies are presented in $\mathrm{eV}$.

electron density and an idealised bulk electron density with the same number of electrons, but no relaxation of the density into the vacuum. The effect of the dipole across the surface-resulting from the spilling of electron density—on the electrostatic potential is then calculated from the Poisson equation

$$
\nabla^{2} V=-\frac{\rho_{\text {planar }}}{\epsilon},
$$

where $\rho_{\text {planar }}$ is the difference between the truncated bulk electron density and the slab electron density, $\mathrm{V}$ is the electrostatic potential, and $\epsilon$ is the dielectric constant of the medium.

The results are presented in Figure 2, where the charge density of the slab and the difference between the slab and the resultant electrostatic potential are plotted. We consider the pristine surface and the reduced surface $\left(\mathrm{Mo}_{10} \mathrm{O}_{28}\right)$, where the top layer of oxygens have been removed, allowing us to evaluate the effects of the surface structure on the electron energies.

For the pristine surface there is a net spilling of electrons out from the surface and the dipole calculated by applying Equation (2) is $3.3 \mathrm{~V}$, a stabilisation of electrons in the slab. For the reduced slab the situation is quite different; there is a significantly less pronounced spill-out of electron density. This is because the excess electron becomes localised on the 


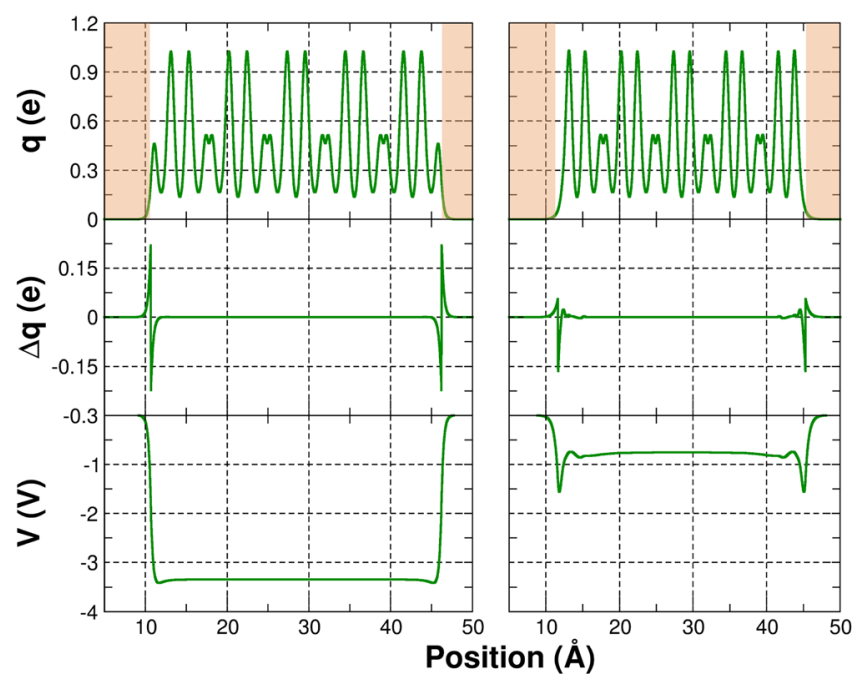

FIG. 2. The effect of the surface dipole on slab electrostatic potential; left, pristine surface and right, reduced surface. Upper panel shows the slab planar electron density $(q)$, middle panel shows the surface double-layer $(\delta q)$, and the lower panel shows the resultant electrostatic potential (V). The slab thickness is $\sim 30 \AA$, and the slabs are centered on the abscissa; the vacuums are $\sim 20 \AA$.

Mo, reducing the oxidation state. The surface dipole is $0.8 \mathrm{~V}$, a smaller stabilisation of the electrons in the slab. This difference in surface electrostatic contribution of $2.5 \mathrm{~V}$ explains the large difference in the IPs and EAs between the pristine and reduced slabs $\left(\mathrm{MoO}_{x}\right.$ and $\mathrm{MoO}_{3}$ in Figure 1). Note that these IP and EA values are for the bulk of the material, far from the surface. However, the excess of electrons associated with the reduction can also effectively $n$-type dope the material, as indicated by the shading in the conduction band of $\mathrm{MoO}_{x}$ in Figure 1.

Water molecules adsorbing on the surface of the $\mathrm{MoO}_{3}$ are considered by placing $0.06,0.22,0.5$, and 1.0 monolayers (ML) on the surface and geometry optimising the system. The water molecules carry a permanent dipole, which may be expected to affect the ionization potential of the slab by modifying the $\Delta V_{\text {surf }}$ term in Equation (1). The influence of this dipole depends on the distance and orientation of the double layer from the surface. ${ }^{22}$ We therefore begin by considering how the water arranges itself on the surface. In this case a $3 \times 3$ supercell expansion of the surface and $\Gamma-$ point sampling of $k$-space was used.

As was reported previously, ${ }^{39}$ the most stable position for a single water molecule per oxygen on the surface is above the protruding oxygen (note that we refer to this as 0.5 ML coverage, whilst reference 43 refers to it as $1.0 \mathrm{ML}$ coverage), and this corresponds to one water per protruding oxygen, as indicated by the jade squares in Figure 3(d). When the coverage of the surface is less than 0.5 ML (i.e., 0.06 ML and $0.22 \mathrm{ML}$ ) the position of water adsorption changes; the preferred site at lower concentrations is the bridging oxygen (the white squares in Figure 3(d)). This difference in bonding location is reflected by a shorter water oxygen to surface oxygen separation at lower coverage concentration (Figure 3(a)). In the bridging conformation the water molecules are h-bonded to two surface oxygens, whereas at $0.5 \mathrm{ML}$ in the on top conformation the water is H-bonded to one oxygen and has a separation of the hydrogen in one water to the oxygen in the next of $\sim 2.8 \AA$, suggesting that the oxygen lone pair and the hydrogen could form a weak hydrogen bond. At 1.0 ML coverage the situation is very different. After all protruding oxygens are occupied, the next most stable site is above the bridging oxygen of the surface. We find that the next layer of water adsorbs here and form a $\mathrm{H}$ bonded network with the other $\mathrm{H}_{2} \mathrm{O}$ molecules on the surface.

The angle between the permanent dipole of each water molecule and the plane of the surface remains largely constant over up to $0.5 \mathrm{ML}$ of coverage at $\sim 87^{\circ}$. From classical electrostatics we would therefore expect that as coverage increases the strength of the dipole contribution is additive. ${ }^{22}$ However, the water adsorption also affects the double layer of the slab (discussed earlier). This is evidenced by the striking observation (Figure 3(c)) that below 0.5 ML the water acts to decrease the IP and above $0.5 \mathrm{ML}$ it acts to increase the IP. This means that the concentration of water adsorbed at the surface will be decisive in determining how the energy levels of the HTL are affected by atmospheric moisture, with consequences in terms of band-alignment in device architectures.

Another important effect of $\mathrm{H}_{2} \mathrm{O}$ is the break-up and formation of small clusters of $\mathrm{MoO}_{3}$, which occurs by intercalation of water molecules between $\mathrm{MoO}_{3}$ layers. ${ }^{40}$ Gwinner et al. demonstrate the effect of this breakup and clustering on the energy levels of the oxide. We have used the experimental values ${ }^{19}$ to populate our energy-band-alignment diagram as " $\mathrm{MoO}_{3}$ cluster." Here the bandgap widens and the band edges move closer to the vacuum level.

A water layer at the surface of $\mathrm{MoO}_{3}$ has also been shown to result in a change in the oxidation state of Mo from +6 to $+5,{ }^{14}$ which has been associated with a concomitant opening of the bandgap. Two possible mechanisms for reduction of $\mathrm{Mo}$ by $\mathrm{H}_{2} \mathrm{O}$ are the removal of the surface $\mathrm{O}$ by hydrolysis or the hydroxylation of the surface $\mathrm{O}$. The former scenario results in " $\mathrm{MoO}_{x}$ " in Figure 1, and the latter is " $\mathrm{MoO}_{3} \mathrm{OH}$ " in the same diagram. As we demonstrated earlier, the reduction of the surface results in a reduced surface dipole and therefore a shallower IP.

Hydroxylation has a similar effect on the IP and EA to the surface reduction. In $\mathrm{MoO}_{3} \mathrm{OH}$ the band gap has been observed to increase. ${ }^{14,19}$ In this case the reduction of the surface results in additional charge being transferred to the $\mathrm{MoO}_{3}$ layer. The previously unoccupied d-orbitals of the $\mathrm{MoO}_{3}$ are now occupied, resulting in a degenerate doping regime with the Fermi level in the conduction band and a Moss-Burstein shift of the measured optical bandgap; ${ }^{41}$ therefore, our calculations are consistent with experimental observations and we conclude that this is a possible mechanism for the observed reduction of Mo.

Previously we reported how, using thermally annealed $\mathrm{MoO}_{x}$, solar cell performance could be improved by air processing as opposed to processing in an inert atmosphere. ${ }^{18}$ These findings can be broadly aligned with the results of the materials modelling presented above, which suggest a complex interplay of effects of $\mathrm{H}_{2} \mathrm{O}$ incorporation at TMO HTLs. Microscopic factors such as improved doping of the organic layer and increased Fermi level of the oxide can lead to an improvement in some aspects of device performance at 


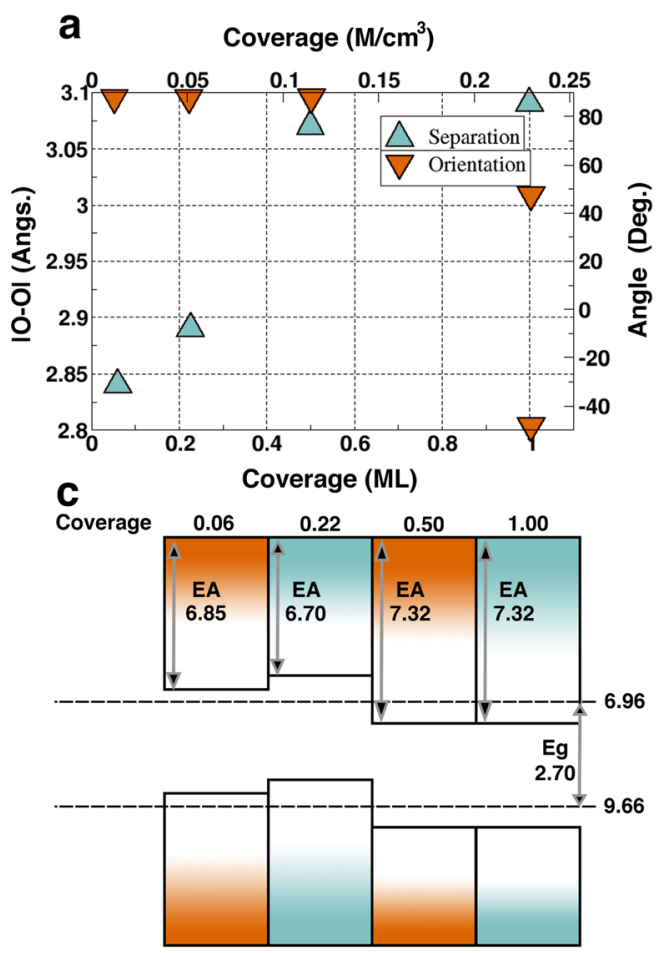

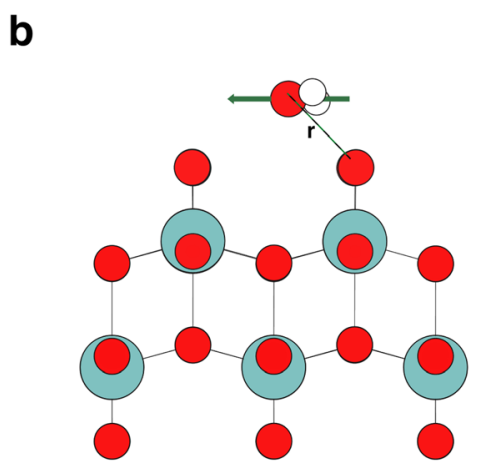

d

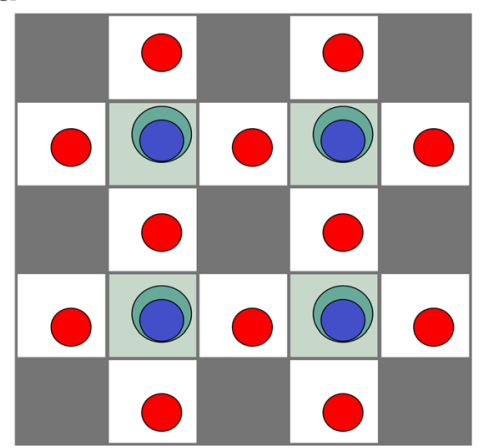

FIG. 3. Properties of the hydrated surfaces of $\mathrm{MoO}_{3}$. (a) Angles (grey) made by the water dipole to the surface normal vector and separations (orange) of adsorbed water with respect to the $\mathrm{MoO}_{3}$ surface as a function of coverage. (b) Representative geometry of water adsorbed to the surface showing the direction of the water dipole (green arrow) and separation distance as plotted in (a). (c) Energy-band-alignment diagram of $\mathrm{MoO}_{3}$ with water coverage; the dashed horizontal lines show the energy levels of the pristine material. (d) Chequerboard representation of surface adsorption sites. Green circles are Mo, blue circles are protruding surface $\mathrm{O}$, and red circles are bridging surface oxygens, jade squares represent on-top adsorption sites, white squares represent bridging oxygen adsorption sites, and grey squares are dead-zone non-interacting sites. well-defined levels of surface hydration. On the other hand, effects such as fragmentation of the TMO lead to diminished performance.

We note that water can also play an important role in determining the morphology and wetting behavior of HTLs and absorber layers. It has been demonstrated that surface modification of the HTL can alter interface wetting behavior and alter photovoltaic performance. ${ }^{42}$ It has also recently been shown in hybrid organic-inorganic materials that small amounts of water can promote crystallisation of the absorber layer. ${ }^{43}$ The study of these morphological effects is beyond the scope of the current study; nonetheless, this important consideration should be borne in mind when considering potential effects of atmosphere on device performance.

In conclusion we have studied the effects of altering the surface of $\mathrm{MoO}_{3}$ by the presence of ambient moisture. We demonstrate how the surface double layer can affect the IP; altering the oxidation of the surface changes the double-layer and shifts the IP by almost $2.5 \mathrm{eV}$. We show how the adsorption of water on the surface can affect the surface polarisation. We find that low concentrations of water $(<0.5 \mathrm{ML})$ reduce the IP while higher concentrations $(>0.5 \mathrm{ML})$ increase the IP. We also explore the effects of surface hydroxylation, which, like surface reduction, can shift the IP closer to the vacuum level. These findings provide guidelines and emphasise the importance of process optimisation to optimise the electronic properties of the final device architecture.

We acknowledge J. M. Frost for insightful discussions regarding organic photovoltaic device characteristics. We acknowledge support from the EPSRC (Grants EP/M009580/1, $\mathrm{EP} / \mathrm{J} 017361 / 1, \mathrm{EP} / \mathrm{I01330X/1,} \mathrm{and} \mathrm{EP/I028641/1),} \mathrm{the} \mathrm{Royal}$ Society, and the European Research Council. The work benefited from the University of Bath's High Performance Computing Facility, and access to the HECToR supercomputer through membership of the UKs HPC Materials Chemistry Consortium, which is funded by EPSRC (Grant No. EP/ F067496) and the UltraFOx grant.

${ }^{1}$ Z.-K. Tan, Y. Vaynzof, D. Credgington, C. Li, M. T. L. Casford, A. Sepe, S. Huettner, M. Nikolka, F. Paulus, L. Yang, H. Sirringhaus, N. C. Greenham, and R. H. Friend, Adv. Funct. Mater. 24, 3051 (2014).

${ }^{2}$ Y. Zhou, C. Fuentes-Hernandez, J. Shim, J. Meyer, A. J. Giordano, H. Li, P. Winget, T. Papadopoulos, H. Cheun, J. Kim, M. Fenoll, A. Dindar, W. Haske, E. Najafabadi, T. M. Khan, H. Sojoudi, S. Barlow, S. Graham, J.-L. Brédas, S. R. Marder, A. Kahn, and B. Kippelen, Science 336, 327 (2012).

${ }^{3}$ K. T. Butler and J. H. Harding, Phys. Rev. B 86, 245319 (2012).

${ }^{4}$ K. T. Butler, Phys. Status Solidi A 212, 1461 (2015).

${ }^{5}$ M. Muccini, Nat. Mater. 5, 605 (2006).

${ }^{6}$ S. Liu, R. Liu, Y. Chen, S. Ho, J. H. Kim, and F. So, Chem. Mater. 26, 4528 (2014).

${ }^{7}$ F. Xie, W. C. H. Choy, C. Wang, X. Li, S. Zhang, and J. Hou, Adv. Mater. 25, 2051 (2013).

${ }^{8}$ X. Li, F. Xie, S. Zhang, J. Hou, and W. C. H. Choy, Adv. Funct. Mater. 24, 7348 (2014).

${ }^{9}$ P. Qin, G. Fang, F. Cheng, W. Ke, H. Lei, H. Wang, and X. Zhao, ACS Appl. Mater. Interfaces 6, 2963 (2014).

${ }^{10}$ M. D. Irwin, D. B. Buchholz, A. W. Hains, R. P. H. Chang, and T. J. Marks, Proc. Natl. Acad. Sci. U. S. A. 105, 2783 (2008).

${ }^{11}$ P. Schulz, S. R. Cowan, Z.-L. Guan, A. Garcia, D. C. Olson, and A. Kahn, Adv. Funct. Mater. 24, 701 (2014).

${ }^{12}$ T. Aytun, A. Turak, I. Baikie, G. Halek, and C. W. Ow-Yang, Nano Lett. 12, 39 (2012).

${ }^{13}$ M. T. Greiner, M. G. Helander, Z. B. Wang, W. M. Tang, J. Qiu, and Z. H. Lu, Appl. Phys. Lett. 96, 213302 (2010).

${ }^{14}$ J. Meyer, S. Hamwi, M. Kröger, W. Kowalsky, T. Riedl, and A. Kahn, Adv. Mater. 24, 5408 (2012).

${ }^{15}$ A. A. Balandin and I. D. Rozhdestvenskaya, Bull. Acad. Sci. USSR, Div. Chem. Sci. 8, 1804 (1959).

${ }^{16}$ J. Griffin, D. C. Watters, H. Yi, A. Iraqi, D. Lidzey, and A. R. Buckley, Adv. Energy Mater. 3, 903 (2013).

${ }^{17}$ T. A. Papadopoulos, J. Meyer, H. Li, Z. Guan, A. Kahn, and J.-L. Brédas, Adv. Funct. Mater. 23, 6091 (2013).

${ }^{18}$ E. S. R. Bovill, J. Griffin, T. Wang, J. W. Kingsley, H. Yi, A. Iraqi, A. R. Buckley, and D. G. Lidzey, Appl. Phys. Lett. 102, 183303 (2013).

${ }^{19}$ M. C. Gwinner, R. D. Pietro, Y. Vaynzof, K. J. Greenberg, P. K. H. Ho, R. H. Friend, and H. Sirringhaus, Adv. Funct. Mater. 21, 1432 (2011). 
${ }^{20}$ S. W. Cho, L. F. J. Piper, A. DeMasi, A. R. H. Preston, K. E. Smith, K. V. Chauhan, R. A. Hatton, and T. S. Jones, J. Phys. Chem. C 114, 18252 (2010).

${ }^{21}$ K. T. Butler, J. Buckeridge, C. R. A. Catlow, and A. Walsh, Phys. Rev. B 89, 115320 (2014).

${ }^{22}$ K. T. Butler and A. Walsh, Thin Solid Films 559, 64 (2014).

${ }^{23}$ G. Heimel, F. Rissner, and E. Zojer, Adv. Mater. 22, 2494 (2010).

${ }^{24}$ D. O. Scanlon, G. W. Watson, D. J. Payne, G. R. Atkinson, R. G. Egdell, and D. S. L. Law, J. Phys. Chem. C 114, 4636 (2010).

${ }^{25}$ R. Coquet and D. J. Willock, Phys. Chem. Chem. Phys. 7, 3819 (2005).

${ }^{26}$ G. Kresse and J. Furthmüller, Phys. Rev. B 54, 11169 (1996).

${ }^{27}$ P. E. Blöchl, Phys. Rev. B 50, 17953 (1994).

${ }^{28}$ G. Kresse, Phys. Rev. B 59, 1758 (1999).

${ }^{29}$ L. Kihlborg, Ark. Kemi 21, 357 (1963).

${ }^{30}$ J. P. Perdew, A. Ruzsinszky, G. I. Csonka, O. A. Vydrov, G. E. Scuseria, L. A. Constantin, X. Zhou, and K. Burke, Phys. Rev. Lett. 100, 136406 (2008).

${ }^{31}$ J. Moreno and J. M. Soler, Phys. Rev. B 45, 13891 (1992).

${ }^{32}$ A. V. Krukau, O. A. Vydrov, A. F. Izmaylov, and G. E. Scuseria, J. Chem. Phys. 125, 224106 (2006).
${ }^{33}$ See https://github.com/WMD-Bath/MacroDensity for information on obtaining Hartree potential plots (accessed July 30, 2015).

${ }^{34}$ M. Kroger, S. Hamwi, J. Meyer, T. Riedl, W. Kowalsky, and A. Kahn, Appl. Phys. Lett. 95, 123301 (2009).

${ }^{35}$ J. Bardeen, Phys. Rev. 49, 653 (1936).

${ }^{36}$ N. Lang and W. Kohn, Phys. Rev. B 3, 1215 (1971).

${ }^{37}$ A. J. Logsdail, D. O. Scanlon, C. R. A. Catlow, and A. A. Sokol, Phys. Rev. B 90, 155106 (2014).

${ }^{38}$ J. Buckeridge, K. T. Butler, C. R. A. Catlow, A. J. Logsdail, D. O. Scanlon, S. A. Shevlin, S. M. Woodley, A. A. Sokol, and A. Walsh, Chem. Mater. 27, 3844 (2015).

${ }^{39}$ X. Yin, H. Han, and A. Miyamoto, Mol. Mod. Ann. 7, 207 (2001).

${ }^{40}$ A. Kuzmin and J. Purans, J. Phys.: Condens. Matter 12, 1959 (2000).

${ }^{41}$ M. Grundmann, The Physics of Semiconductors, 2nd ed. (Springer-Verlag, Berlin Heidelberg, 2010).

${ }^{42}$ N. E. Widjonarko, P. Schulz, P. A. Parilla, C. L. Perkins, P. F. Ndione, A. K. Sigdel, D. C. Olson, D. S. Ginley, A. Kahn, M. F. Toney, and J. J. Berry, Adv. Energy Mater. 4, 1301879 (2014).

${ }^{43}$ K. K. Bass, R. E. McAnally, S. Zhou, P. I. Djurovich, M. E. Thompson, and B. C. Melot, Chem. Commun. 50, 15819 (2014). 